\title{
Six-week physical rehabilitation protocol for anterior shoulder dislocation in athletes
}

\author{
Ahmed Gaballah ${ }^{1,2 *}$, Mohamed Zeyada ${ }^{3}$, Adham Elgeidi', Eadric Bressel' \\ 'Department of Kinesiology and Health Science, Utah State University, Logan, UT, USA \\ ${ }^{2}$ Department of Sports Health Science, Damietta University, Damietta, Egypt \\ ${ }^{3}$ Department of Sports Health Science, Mansoura University, Mansoura, Egypt \\ ${ }^{4}$ Department of Orthopaedic Surgery, Mansoura University, Mansoura, Egypt
}

Anterior shoulder dislocations are common in young athletes. The mechanism for the first or primary shoulder dislocation may involve a collision or a fall typically with the arm in an abducted and externally rotated position. The aim of this study was to design a physical rehabilitation program using the elastic band and resistive exercise to improve joint strength and range of motion in individuals diagnosed with a firsttime shoulder dislocation. Twelve physically active males with a firsttime acute shoulder dislocation were asked to volunteer. Participants began a physical rehabilitation program 2 weeks after the shoulder dislocation, which was confirmed by a referring physician. The rehabilitation program was 6 weeks in duration and required the participants to engage in progressive resistive loads/duration using elastic bands and weights 5 days per week. Pretest and posttest measures included shoulder strength and range of motion. All outcome measures were compared between the injured and uninjured shoulder, which served as the control condition in this study. There were statistically significant differences between the injured and uninjured shoulder for measures of strength and range of motion during pretests $(P<0.01)$ but not posttests $(P<0.53)$. Finally, there were no differences between shoulders in regards to the volume measure suggesting that any changes in muscle atrophy or swelling were not detected. The physical rehabilitation program proposed in this study was effective at improving strength and range of motion in the injured shoulder as evidenced by the similarity in posttest values between the injured and uninjured shoulder.

Keywords: Shoulder dislocation, Elastic resistant exercises, Physical rehabilitation, Shoulder injuries

\section{INTRODUCTION}

The shoulder is the most frequently dislocated joint in the human body, anterior dislocation is the most common injury in our daily life, especially for young people (Liu et al., 2014; Rumian et al., 2011). It was estimated that the incidence rate of shoulder dislocation is 23.9 per 100,000 persons a year (Owens et al., 2009). Anterior shoulder dislocations and subluxations are common injuries in young athletes (Kaplan et al., 2005; Malhotra et al., 2012). The disparity between the large humeral head and the small glenoid cavity increasing the joint ability to be injured (Brandt et al., 2013; Brukner and Kham, 2012). Moreover, the anteriorly dislocated humeral head causes a labrum tear of the an-

terior and inferior labrum, a Bankart injury (Longo et al., 2014; Porcellini et al., 2009), and a typical impression fracture (Hovelius et al., 2008; Kim et al., 2003).

The mechanism of the first dislocations occurs after a forceful direct trauma or a fall typically with the arm in an abducted and externally rotated or outstretched arm (Owens et al., 2010). Thus, in the majority of cases, the arm is abducted and the shoulder is externally rotated (Hardy et al., 2010). This is common in rugby, hockey, Handball, Football, riding, and cycling. In patients with lax shoulders or previous dislocations, dislocation can occur after much less trauma (Badr and Gaballah, 2015; Brooks et al., 2005; Kelly and Terry, 2001). Recently, the most common management of treatment for primary anterior glenohumeral instability is

*Corresponding author: Ahmed Gaballah (D) http://orcid.org/0000-0001-8730-3984

This is an Open Access article distributed under the terms of the Creative Commons Attribution Non-Commercial License (http://creativecommons.org/licenses/by-nc/4.0/) which permits unrestricted non-commercial use, distribution, and reproduction in any 7000 Old Main Hill Logan, UT 84322-7000, USA

Tel: +1-435-557-6699, Fax: +1-435-797-3759, medium, provided the original work is properly cited.

E-mail: ahmed.gaballah@aggiemail.usu.edu

Received: April 19, 2016 / Accepted: June 10, 2017 
non-operative management (Gibson et al., 2004; Zacchilli and Owens, 2010). Rehabilitation and prevention of this injury are worthwhile of research because the negligence of rehabilitation for the first-time dislocation shoulder may lead to recurrent instability of the glenohumeral joint (Provencher et al., 2010; Rumian et al., 2011). Furthermore, there is a body of reports suggesting that younger athletes generally and contact sports particularly are most affected with an instability risk after a primary anterior shoulder dislocation (Handoll et al., 2006; Zacchilli and Owens, 2010). This risk of the recurrence dislocation reported in young athletes with first-time anterior dislocation is high as 90\%-95\% (Gibson et al., 2016). However, the athletes with anterior shoulder dislocation who returned to activities within the season after lack rehabilitation have demonstrated high rates of recurrent dislocation from 37\% to 90\% (Castagna et al., 2007; Watson et al., 2016). The goal of the primary rehabilitation for the acute anterior shoulder is to prevent long-term instability for the shoulder joint (Leroux et al., 2014). However, patients with recurrent shoulder instability often need a surgical intervention or arthroscopic treatment (Malhotra et al., 2012; Porcellini et al., 2009; Provencher et al., 2010). Indeed, several modalities used conservative treatment for the acute dislocation shoulder (Liavaag et al., 2011; Liu et al., 2014; Yamamoto et al., 2010) and injections (Nagata et al., 2015). Some approaches have conducted physical rehabilitation programs for a first-time shoulder dislocation (Gibson et al., 2004; Karatsolis and Athanasopoulos, 2006; Salamh and Speer, 2013). Other used the elastic resistance exercise which has become one of the popular tools in physical rehabilitation (Andersen et al., 2010; Brandt et al., 2013; Camci et al., 2013). The major reason to use the elastic band is that they have demonstrated excellent validity and reliability with shoulder muscle testing (Andersen et al., 2016). Ultimately, the aim of this study is to design a physical rehabilitation program using an elastic band and resistive exercise to improve joint strength in individuals diagnosed with a firsttime shoulder dislocation.

\section{MATERIALS AND METHODS}

\section{Subjects}

Physically active males who came to Mansoura University Hospitals and the teaching hospital in Damietta, Egypt with acute anterior dislocation shoulder injury between September 2013 and February 2016 were randomly recruited as research participants. Magnetic resonance imaging was conducted to determine the injury grade for 17 athletes. Furthermore, excluded the patients with

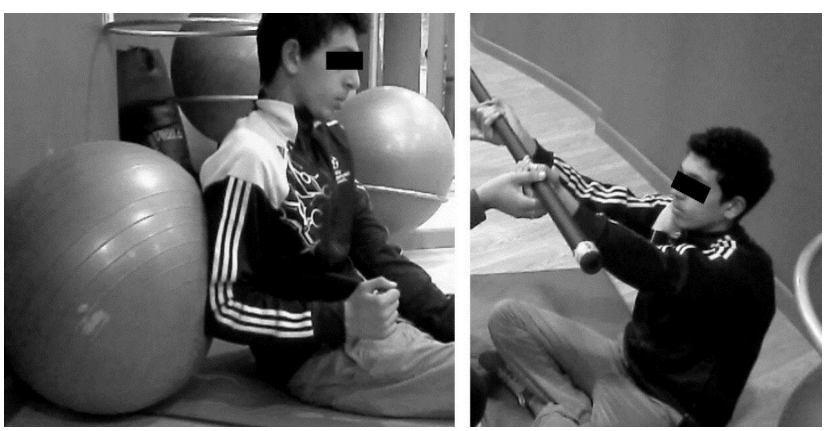

Fig. 1. Isometric exercises examples in the first rehabilitation stage.

recurrent dislocation shoulder or with a history of injury in the same joint. Twelve physically active males (age, 18.6 \pm 1.32 years; mass, $74.48 \pm 3.22 \mathrm{~kg}$; height, $178.4 \pm 3.21 \mathrm{~cm}$ with competitive experience $9.6 \pm 2.67$ years) and first-time acute shoulder dislocation were considered as research participants. The participants began a physical rehabilitation program 2 weeks after the shoulder dislocation, which was confirmed by a referring physician.

\section{Rehabilitation program}

The duration of the rehabilitation protocol was 6 weeks and was divided into three stages. However, the duration of each stage was 2 weeks. Seventeen external and internal rotation exercises were chosen for the first stage which aimed to control the pain and inflammation caused by the dislocation. Also, was included flexibility and isotonic strength exercise with $12-15$ reps and 30\% intensity of one-repetition maximum (1RM) for the healthy shoulders. Which performed for the scapulothoracic muscles, in particular, the rotator cuff muscles (Fig. 1). The second stage aimed to restore the higher level of muscle strength. The intensity of exercises was five sets with 8-10 reps and 60\%-70\% of $1 \mathrm{RM}$ for the healthy shoulders. Thirty-two exercises were performed in the second stage. In particular, Deltoid M., Trapezius M., and Serratus Anterior M. exercises in this stage were performed widely in the horizontal and diagonal axes. Moreover, the exercises were chosen to enhance the range of motion (ROM) and muscles strength between $90^{\circ}-150^{\circ}$ vertically, horizontally, and diagonally (Fig. 2).

The third stage was designed for the endurance, plyometric, and strength exercises and was consisted of five sets of with 3-6 reps using variable resistance: one at $75 \%$ of the 10RM, and two at $95 \%$ of the 1RM. Twenty-seven extremity weights rehabilitation exercises were performed to reach $190^{\circ}-200^{\circ} \mathrm{ROM}$ and the equally healthy arms strength as well (Fig. 3). The Thera-Band resistance exercises were used during the six weeks especially the four colors (red, blue, black, silver, and gold). The red and blue 

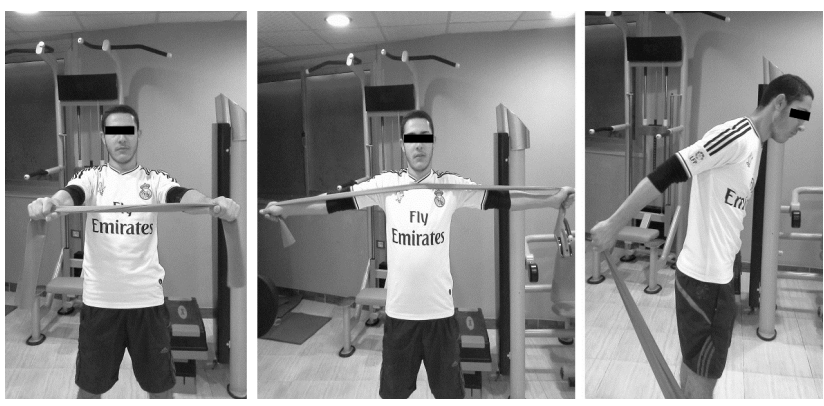

Fig. 2. Elastic band exercises examples in the second rehabilitation stage.
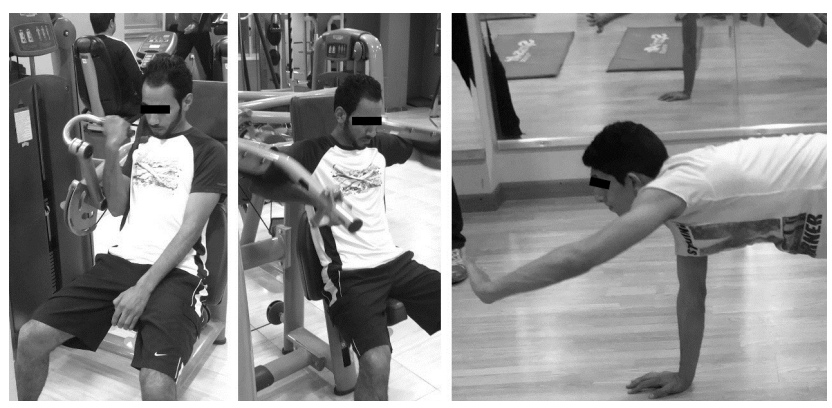

Fig. 3. Endurance and strength exercises examples in the third rehabilitation stage.

bands are used in the first stage and the black and silver used in the second stage, In addition, the gold was used in the third stage. Furthermore, all the exercises performed by stretching the band between 75\%-100\%. knowing that, the weight of stretching the Thera-Band between 75\%-100\% is (red, 3.3-3.9; blue, 5.9-7.1; black, 8.1-9.7; silver, 11.1-13.2; and gold, 18.1-21.6 kg) (Andersen et al., 2010; BiÇer et al., 2015).

\section{Data collection}

The rehabilitation program comprised four testing sessions. The pretest section was conductedth after 2 weeks of the injury and before the beginning of the rehabilitation program. After two rehabilitative weeks, the 2 nd-week tracking test was realized, while the 4th-week tracking test was realized after the 4th rehabilitative week. Moreover, after a resting of six weeks, posttest was collected. Additionally, all the injured athletes were right handed and in each test, the healthy arms measurements were recorded. Continuously, the test results for the injured arm had been compared with the healthy arm for each person. Before reporting the force measurement, all the participants were informed of the test procedures for both arms. Four basics Isotonic force measurements were reported by an Isometric Dynamometer device (Isokinetic Dynamometer, BiodexMedical Systems, Shirley, NY, USA). These

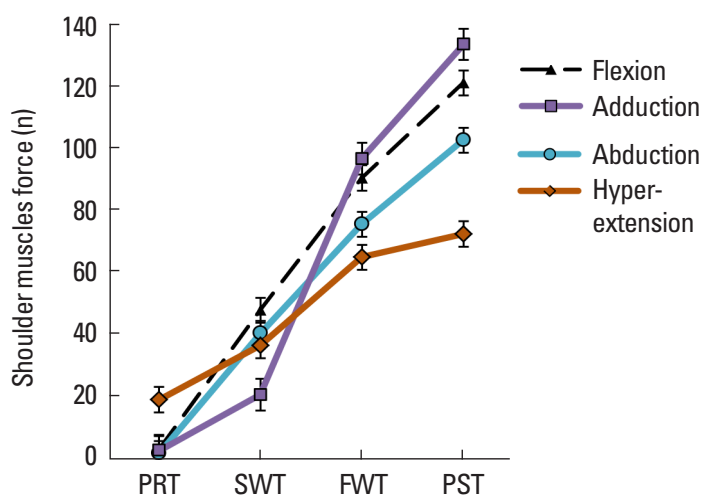

Fig. 4. The improvement of shoulder muscles strength after the exercise protocol for the injured arms. PRT, pretest; SWT, 2nd week test; FWT, 4th week test; PST, posttest.

force measurements were conducted for muscles around shoulder girdle in the $90^{\circ}$, vertically and horizontally. The force variables were flexion force (FF), adduction force (ADF), abduction force $(\mathrm{ABF})$, and hyperextension force (HBF). The participants were asked to perform the maximum flexibility for the shoulder joint without feeling pain. In addition, the ROM variables were obtained by goniometer for flexion ROM (F.ROM), abduction ROM (AB.ROM), and hyper extension ROM.

\section{Data analyze}

The paired $t$-test was used to compare the collected data before performing the exercise protocol (pretest) and those which were obtained after 6-week physical rehabilitation period (posttest). All the analyses were performed by using IBM SPSS Statistics ver. 19.0 (IBM Co., Armonk, NY, USA). Additionally, all values within the text and table are observed as standard deviation and mean.

\section{RESULTS}

The test results (i.e., strength and ROM) show that there were no statistically significant differences between the injured and uninjured shoulder $(P>0.01)$ prior to the rehabilitation procedure. After 6-week physical rehabilitation, the test results showed considerable improvement of more than $90 \%$. The difference between the pretestes and posttests of force were, FF $4.41 \pm 4.01$ vs. 121.5 $\pm 12.4, \mathrm{ABF} 2.94 \pm 2.74$ vs. $103.1 \pm 9.8, \mathrm{ADF} 3.84 \pm 0.34$ vs. $133.7 \pm 9.26$, $\mathrm{HBF} 20.01 \pm 7.64$ vs. $69.1 \pm 8.92$ (Fig. 4). On the other hand, the ROM of pretestes and posttest were, FF $23.8^{\circ} \pm 4.02^{\circ}$ vs. $199.5^{\circ} \pm 0.24^{\circ}, \mathrm{ABF}$ was $29.3^{\circ} \pm 3.62^{\circ}$ vs. $195.5^{\circ} \pm 2.13^{\circ}, \mathrm{HBF} 30.8^{\circ} \pm 5.81^{\circ}$ vs. $108.5^{\circ} \pm 5.72^{\circ}$. Furthermore, the conducted post tests have shown that there were no sig- 


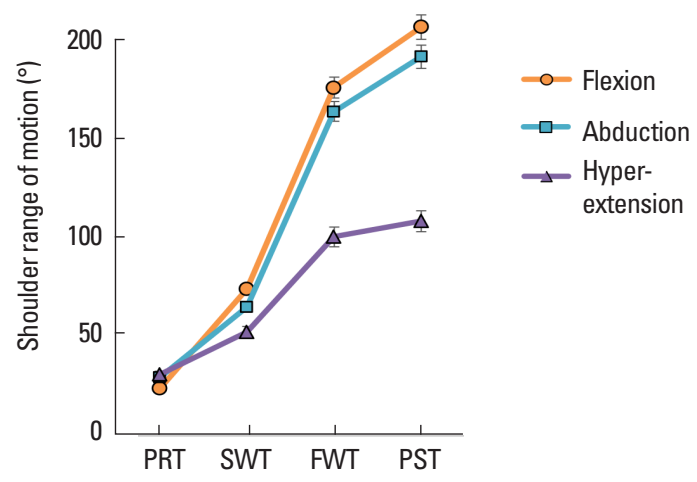

Fig. 5. The improvement of shoulder range of motion after the exercise protocol for the injured arms. PRT, pretest; SWT, 2nd week test; FWT, 4th week test; PST, posttest.

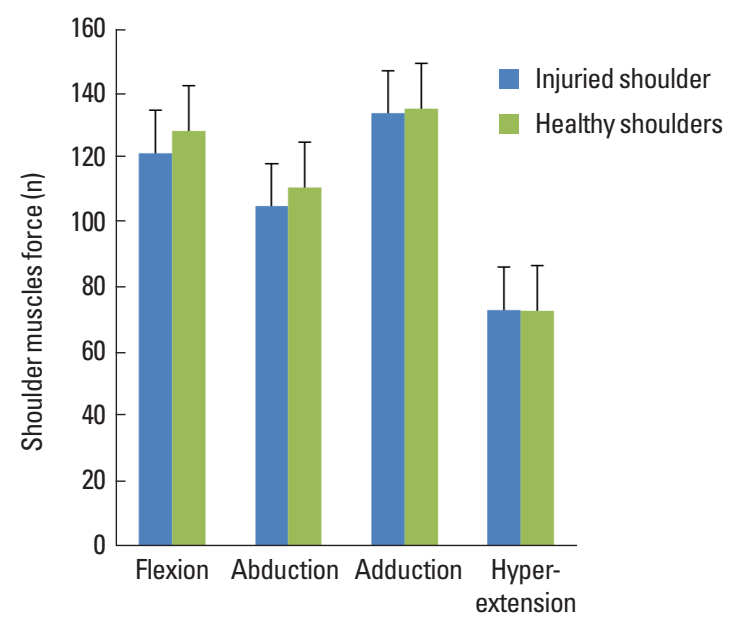

Fig. 6. The posttests difference in shoulder muscle strength between the injured and healthy arms.

nificant differences $(P<0.53)$ between the shoulder muscle force for the injured and uninjured shoulders. However, the participants' injured shoulder showed great improvement after the rehabilitation (Fig. 5).

In addition to the posttests of the injured compared with the uninjured shoulders were, FF $121.5 \pm 12.44$ vs. $128.2 \pm 10.4$ with $94 \%$ enhancement, $\mathrm{ABF} 103.1 \pm 9.81$ vs. $110.9 \pm 8.32$ was $93 \%$ enhancement, ADF $133.7 \pm 9.26$ vs. $135.2 \pm 5.33$ with enhancement $99 \%$, HBF $69.1 \pm 8.92$ vs. $72.71 \pm 5.42$ with $95 \%$ enhancement (Fig. 6). In contrast, F.ROM $199.5^{\circ} \pm 0.24^{\circ}$ vs. $201.3^{\circ} \pm 1.25^{\circ}$ was $99 \%$ enhancement, AB.ROM $195.5^{\circ} \pm 2.13^{\circ}$ vs. $198.9^{\circ} \pm$ $1.06^{\circ}$ had $98.3 \%$ enhancement, and HE.ROM $107.8^{\circ} \pm 6.12^{\circ}$ vs. $108.5^{\circ} \pm 5.72^{\circ}$ was $99.4 \%$ enhancement (Fig. 7). Finally, the rehabilitation did not induce any side effects: muscle atrophy changes and swells were not detected.

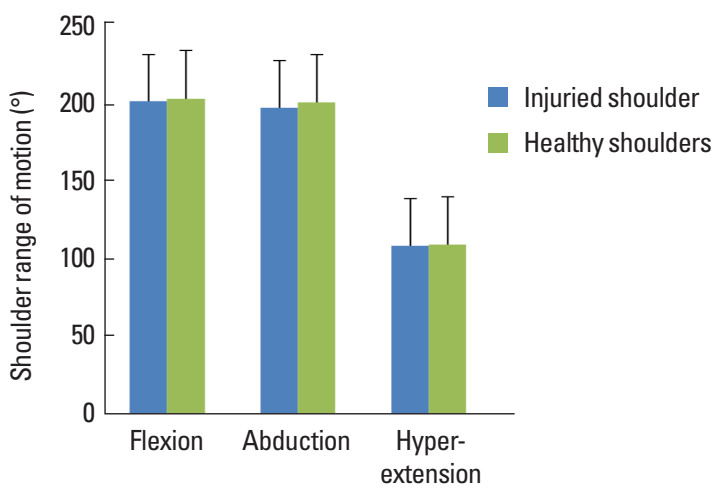

Fig. 7. The posttests difference in shoulder range of motion between the injured and healthy arms.

\section{DISCUSSION}

The current study aimed to design a rehabilitation program used the elastic and weights exercises. Over the past decades, we have seen a huge boost in the rehabilitation of shoulder dislocation injuries. These injuries occurred because of the anatomical structure of the glenohumeral joint. Therefore, derives its extensive movement at the expense of its consistency and coherence. More than $50 \%$ of acute anterior dislocations can reoccur, especially in young athletes. The aim of the physical rehabilitation is avoiding the recurrent dislocation and using an elastic band and resistive exercise to improve joint strength in individuals diagnosed with a first-time shoulder dislocation.

The current study results show that there was weakness of the shoulder muscles in the pretest measurements of $\mathrm{FF}, \mathrm{ADF}, \mathrm{ABF}$ as a result of pain, swelling, and inflammation. Nevertheless, the hyperextension motion measurements were reported better than the other measurement in the pretest. The mechanism of the anterior dislocation occurred forwardly and downward. Out of the ordinary, that affected adversely on the forward movement of the arm (Turkel et al., 1981)

The return to activity after $2-3$ weeks is available for athletes with a high risk of recurrence dislocation, which compares to arthroscopic remain and return to activity after a 6-month surgical stabilization (Watson et al., 2016). Many factors have been related to rehabilitating shoulder dislocation. Thus, the current study concluded that six weeks' elastic resistant with weight exercise had great benefit for the shoulder muscles especially, the Rotator Cuff muscles. Recently, the popularity of elastic bands has increased because of their low prices, flexibility, adaptability, versatility, and 
simplicity (Andersen et al., 2010; Jakobsen et al., 2013). The incorporation between Nautilus machine and the elastic band's exercises have been used to promote the joint ROM and muscles strength in training and rehabilitation purposes as well. Thus, the elastic resistant exercise has improved the strength-shorting cycle. In addition, it has been used as a technique to produce the speed eccentric contraction (Stevenson et al., 2010).

It is seen in Figs. 4 and 5. that there is a considerable significant difference between the pretests and the posttests of the injured shoulders. Also, it is seeming to be similar to the healthy shoulder after the six weeks' physical rehabilitation program (Fig. 6). The same resulted reported in a previous study conducted on young handball players and has improved their muscle power, muscle strength and ball speed (Mascarin et al., 2016). Nevertheless, the elastic resistant and free weight exercise improve the dynamic stabilization and joint stability (Andersen et al., 2010; Camci et al., 2013). Thus, the exercise rehabilitation program for the unstable shoulder must include a joint position sensibility and dynamic stabilization to support the functional stability of the shoulder joint and promoted the co-activation of the force couples about the glenohumeral joint to exceptional position of the hummers head in the center of glenoid (Naughton et al., 2005).

Previous studies have demonstrated same levels of muscle activation using elastic resistance compared with free weights and display similar or even greater levels of muscle activity for some muscles during an exercise (Andersen et al., 2010; Camci et al., 2013). In contrast, used with the free weights (Aboodardaa et al., 2013). Other studies have shown high levels of muscle activation compared with weight machine (Brandt et al., 2013). But all the modalities recommended use of the elastic band with the weights. Moreover, using the elastic band with weights appear to be equally in muscle activity as a traditional use of isoinertial resistance (Jakobsen et al., 2013). Finally, the physical rehabilitation program proposed in this study was effective at improving strength and ROM in the injured shoulder as evidenced by the similarity in posttest values between the injured and uninjured shoulder. These results are encouraging and suggest the physical rehabilitation program proposed in this study may help reduce the need for surgical intervention in healthy young males who experience a primary shoulder dislocation.

\section{CONFLICT OF INTEREST}

No potential conflict of interest relevant to this article was reported.

\section{ACKNOWLEDGMENTS}

The current study was supported by Faculty of Physical Education, Damietta University, Egypt.

\section{REFERENCES}

Aboodardaa SJ, Hamidb MS, Muhamedc AM, Ibrahim F, Thompsone M. Resultant muscle torque and electromyographic activity during high intensity elastic resistance and free weight exercises. Eur J Sport Sci 2013;13:155-163.

Andersen LL, Andersen CH, Mortensen OS, Poulsen OM, Bjørnlund IB, Zebis MK. Muscle activation and perceived loading during rehabilitation exercises: comparison of dumbbells and elastic resistance. Phys Ther 2010;90:538-549.

Andersen LL, Vinstrup J, Jakobsen MD, Sundstrup E. Validity and reliability of elastic resistance bands for measuring shoulder muscle strength. Scand J Med Sci Sports 2016 May 17 [Epub]. https://doi. org/10.1111/sms.12695.

Badr MA, Gaballah AM. Common injuries among male field hockey players according to playing positions. J Appl Sport Sci 2015;5:19-26.

BiÇer M, Özdal M, Akcan F, MendeȘ B, Patlar S. Effect of strength training program with elastic band on strength parameters. Biol Exerc 2015;11:111-122.

Brandt M, Jakobsen MD, Thorborg K, Sundstrup E, Jay K, Andersen LL. Perceived loading and muscle activity during hip strengthening exercises: comparison of elastic resistance and machine exercises. Int J Sports Phys Ther 2013;8:811-819.

Brooks JH, Fuller CW, Kemp SP, Reddin DB. Epidemiology of injuries in English professional rugby union: part 2 training Injuries. Br J Sports Med 2005;39:767-775.

Brukner P, Khan K, editors. Brukner \& Khan's clinical sports medicine. 4th ed. Sydney: McGraw Hill Medical; 2012.

Camci E, Duzgun I, Hayran M, Baltaci G, Karaduman A. Scapular kinematics during shoulder elevation performed with and without elastic resistance in men without shoulder pathologies. J Orthop Sports Phys Ther 2013;43:735-743.

Castagna A, Nordenson U, Garofalo R, Karlsson J. Minor shoulder instability. Arthroscopy 2007;23:211-215.

Gibson J, Kerss J, Morgan C, Brownson P. Accelerated rehabilitation after arthroscopic Bankart repair in professional footballers. Shoulder Elbow 2016;8:279-286.

Gibson K, Growse A, Korda L, Wray E, MacDermid JC. The effectiveness of rehabilitation for nonoperative management of shoulder instability: a systematic review. J Hand Ther 2004;17:229-242. 
Handoll HH, Hanchard NC, Goodchild L, Feary J. Conservative management following closed reduction of traumatic anterior dislocation of the shoulder. Cochrane Database Syst Rev 2006;(1):CD004962.

Hardy M, Summers D, Edwards J, Munro N, editors. The BMA guide to sports injuries. London: Dorling Kindersley; 2010

Hovelius L, Olofsson A, Sandström B, Augustini BG, Krantz L, Fredin H, Tillander B, Skoglund U, Salomonsson B, Nowak J, Sennerby U. Nonoperative treatment of primary anterior shoulder dislocation in patients forty years of age and younger. a prospective twenty-five-year follow-up. J Bone Joint Surg Am 2008;90:945-952.

Jakobsen MD, Sundstrup E, Andersen CH, Aagaard P, Andersen LL. Muscle activity during leg strengthening exercise using free weights and elastic resistance: effects of ballistic vs controlled contractions. Hum Mov Sci 2013;32:65-78.

Kaplan LD, Flanigan DC, Norwig J, Jost P, Bradley J. Prevalence and variance of shoulder injuries in elite collegiate football players. Am J Sports Med 2005;33:1142-1146.

Karatsolis K, Athanasopoulos S. The role of exercise in the conservative treatment of the anterior shoulder dislocation. J Bodyw Mov Ther 2006;10:211-219.

Kelly L, Terry G. Team handball: shoulder injuries, rehabilitation, and training. Sports Med Arthrosc Rev 2001;9:115-123.

Kim SH, Ha KI, Jung MW, Lim MS, Kim YM, Park JH. Accelerated rehabilitation after arthroscopic Bankart repair for selected cases: a prospective randomized clinical study. Arthroscopy 2003;19:722-731.

Leroux T, Wasserstein D, Veillette C, Khoshbin A, Henry P, Chahal J, Austin P, Mahomed N, Ogilvie-Harris D. Epidemiology of primary anterior shoulder dislocation requiring closed reduction in Ontario, Canada. Am J Sports Med 2014;42:442-450.

Liavaag S, Brox JI, Pripp AH, Enger M, Soldal LA, Svenningsen S. Immobilization in external rotation after primary shoulder dislocation did not reduce the risk of recurrence: a randomized controlled trial. J Bone Joint Surg Am 2011;93:897-904.

Liu A, Xue X, Chen Y, Bi F, Yan S. The external rotation immobilisation does not reduce recurrence rates or improve quality of life after primary anterior shoulder dislocation: a systematic review and meta-analysis. Injury 2014;45:1842-1847.

Longo UG, Loppini M, Rizzello G, Ciuffreda M, Maffulli N, Denaro V. Management of primary acute anterior shoulder dislocation: systematic review and quantitative synthesis of the literature. Arthroscopy 2014;30:506-522.

Malhotra A, Freudmann MS, Hay SM. Management of traumatic anterior shoulder dislocation in the 17- to 25-year age group: a dramatic evolution of practice. J Shoulder Elbow Surg 2012;21:545-553.
Mascarin NC, de Lira CA, Vancini RL, de Castro Pochini A, da Silva AC, Dos Santos Andrade M. Strength training using elastic band improves muscle power and throwing performance in young female handball players. J Sport Rehabil 2016:1-25.

Nagata H, Thomas WJ, Woods DA. The management of secondary frozen shoulder after anterior shoulder dislocation - The results of manipulation under anaesthesia and injection. J Orthop 2015;13:100-105.

Naughton J, Adams R, Maher C. Upper-body wobbleboard training effects on the post-dislocation shoulder. Phys Ther Sport 2005;6:31-37.

Owens BD, Agel J, Mountcastle SB, Cameron KL, Nelson BJ. Incidence of glenohumeral instability in collegiate athletics. Am J Sports Med 2009;37:1750-1754.

Owens BD, Nelson BJ, Duffey ML, Mountcastle SB, Taylor DC, Cameron KL, Campbell S, DeBerardino TM. Pathoanatomy of first-time, traumatic, anterior glenohumeral subluxation events. J Bone Joint Surg Am 2010;92:1605-1611.

Porcellini G, Campi F, Pegreffi F, Castagna A, Paladini P. Predisposing factors for recurrent shoulder dislocation after arthroscopic treatment. J Bone Joint Surg Am 2009;91:2537-2542.

Provencher MT, Bhatia S, Ghodadra NS, Grumet RC, Bach BR Jr, Dewing CB, LeClere L, Romeo AA. Recurrent shoulder instability: current concepts for evaluation and management of glenoid bone loss. J Bone Joint Surg Am 2010;92 Suppl 2:133-151.

Rumian A, Coffey D, Fogerty S, Hackney R. Acute first-time shoulder dislocation. Orthop Trauma 2011;25:363-368.

Salamh PA, Speer KP. Post-rehabilitation exercise considerations following total shoulder arthroplasty. Strength Cond J 2013;35:56-63.

Stevenson MW, Warpeha JM, Dietz CC, Giveans RM, Erdman AG. Acute effects of elastic bands during the free-weight barbell back squat exercise on velocity, power, and force production. J Strength Cond Res 2010;24:2944-2954.

Turkel SJ, Panio MW, Marshall JL, Girgis FG. Stabilizing mechanisms preventing anterior dislocation of the glenohumeral joint. J Bone Joint Surg Am 1981;63:1208-1217.

Watson S, Allen B, Grant JA. A clinical review of return-to-play considerations after anterior shoulder dislocation. Sports Health 2016;8:336341.

Yamamoto N, Sano H, Itoi E. Conservative treatment of first-time shoulder dislocation with the arm in external rotation. J Shoulder Elbow Surg 2010;19(2 Suppl):98-103.

Zacchilli MA, Owens BD. Epidemiology of shoulder dislocations presenting to emergency departments in the United States. J Bone Joint Surg Am 2010;92:542-549. 